\title{
Male and Female Meiosis in Nicotiana tabacum L.
}

\author{
Kuldeep K. Koul* and Ranjna Nagpal \\ Hindu College, Department of Botany, Delhi-110007, India \\ Received April 8, 2004; accepted May 14, 2004
}

\begin{abstract}
Summary Male and female meiosis has been studied together for the first time in Nicotiana tabacum $(2 n=48)$ collected from 2 populations. Meiotic studies revealed differences in the behavior of chromosomes in the 2 sex cells i.e. pollen mother cells (PMCs) and embryo sac mother cells (EMCs) particularly with respect to frequency of chiasmata. Male sex cells (PMCs) showed a higher chiasmata frequency than female sex cells (EMCs) which show 14.33 and $11.48 \%$ reduction in chiasmata frequency in the 2 populations. The practical importance of female meiotic studies is discussed.
\end{abstract}

Key words Nicotiana tabacum, Female meiosis, Chiasmata variation, Genetic control.

Meiosis is a central event which facilitates sexual reproduction and makes the transition from diploid somatic phase to haploid gametic phase possible. From a genetic stand point there are 2 critical events during meiosis. First, members of each homologous pair undergo synapsis. Second, the exchange process referred to as crossing over occurs between synaptic chromosomes. Estimation of cross-over frequencies in any species is of great interest for reasons like 1) they give an idea of homology between various genomes, if present, in any species, 2) they give an impression of overall level of recombination in the species, useful for roughly estimating the size of population required for a reasonable chance to find recombination between desired genes or gene complex, 3) they give an impression of distribution of recombination and, 4) they are basis of chromosome maps showing the relative position of markers and useful genes in linkage groups in chromosomes and the level of recombination between them (Sybenga 1992). Since chiasmata are the cytological manifestation of crossing-over and directly represent the exchange event, they are, therefore, used to estimate the frequency and distribution of crossing-over in various chromosomes. To carry out chiasmata study although 2 types of sex cells are available in the hemaphrodite taxa i.e. pollen mother cell (PMC; male sex cell) and embryo sac mother cell (EMC; female sex cell), it is disheartening to know that whatever meiotic studies have been carried out with a view to study chiasmata frequency, position and distribution, it has been mostly confined to the PMCs only and the information on this aspect in the EMCs has been wanting. Compared to several thousands of plants studied for male meiosis, female meiotic studies have been carried out in some 52 taxa (Fogwil 1958, Ved Brat 1966, Vosa 1972, Bennet et al. 1973, Davies and Jones 1974, Gohil and Kaul 1980, 1981, Kaul 1983, Koul and Gohil 1989, Koul and Raina 1996, Koul and Nagpal 2002, Koul et al. 1995, 1999, 2000). Therefore, it remains largely unknown whether chromosomes in 2 sex cells behave similarly or else show asynchrony in the behaviour with respect to chiasmata position, distribution and frequency. The reasons behind the cytological neglect has been the difficulties faced while locating and handling the delicately placed embryo sac mother cell which is produced in small number, generally, one, per ovule as against the large number of pollen mother cells produced per anther per flower. Owing to difficulties faced in handling female sex cells, extrapolating the behavior of chromosomes in EMCs from the observation made in PMCs has been a common practice which may be misleading. To fill this lacuna research work was initiated in this institution a few years ago and so far sex incidences

* Corresponding author 
of variation in the position, distribution and frequency of chiasmata has been studied in 30 species (Koul et al. 1995, 1999, 2000, Koul and Raina 1996, Koul and Nagpal 2002). Since our earlier studies have revealed drastic differences in the behaviour of chromosomes in the 2 sex cells, it has become mandatory to study a large number of species. Such studies will be important for the complete understanding of genetic/breeding system operational in any species.

In continuation with the work initiated in this institution, presently Tobacco (Nicotiana tabacum L., $2 n=48$ ) plants were studied for male and female meiosis. $N$. tabacum, a member of Solanaceae, is the widely grown non-food crop in the world. Owing to its economic importance although numerous scientific studies have been conducted with this species and much is known about its genetics, physiology and biochemistry (Cameron 1959, Clause and Cameron 1944, Gerstel and Burns 1976, Smith 1979, Reed 1991), the present male and female meiotic studies are being carried out for the first time.

\section{Materials and methods}

Male and female meiosis was studied in Nicotiana tabacum, collected from 2 different populations growing in the Botanical Gardens of Hindu College (HC) and Delhi University (DU). Young unopened buds were fixed in 1:3 acetic alcohol for $24 \mathrm{~h}$ and later preserved in 70\% alcohol till further use. For meiotic studies anthers and ovaries were isolated from the buds using dissection microscope and stained together with leucobasic Fuchsin after hydrolysis in $5 \mathrm{~N} \mathrm{HCl}$ at room temperature for $12 \mathrm{~min}$. Male meiosis was studied by squashing Feulgen stained anthers in a drop of $45 \%$ acetic acid whereas for female meiosis ovules were dissected out of the ovaries in a drop of fixative on a glass slide using long pointed needles. Care was taken to isolate ovules intact. A drop each of $1 \%$ acetocarmine and $45 \%$ acetic acid was placed over the ovule and cover slip was gently lowered on the ovule. After gentle tapping ovules were viewed under the microscope. All the observations were made from the temporary slides and photomicrographs of selected cells were taken using Olympus CH 30 microscope with photomicrographic attachment.

Unlike PMC meiosis which is easy to carry out, serious problems were faced while locating and isolating the EMCs which were generally found hidden under the nucellar tissue and the integuments of the ovules. While separating the EMCs from the surrounding tissue either the EMCs, owing to its bigger size, would break or else chromosomes would scatter all over making the counting of bivalents and the study of chiasmata difficult. This apart, the delicate nature of EMCs also made separation of overlapping bivalents difficult. Despite the various bottlenecks faced while studying the EMC meiosis, 23 and 25 EMCs, with well separated and analyzable bivalents, were studied from the HC and DU populations, respectively. For accurate comparison of chromosome behaviour in the 2 sex cells, the similar meiotic stage i.e. diakinesis, was selected. Statistical methods like Fischer's variance ratio test (F-test) and students $t$-test were applied to determine whether the variations in chiasma frequency in 2 sex cells was significant or not.

\section{Results}

Male and female meiosis were studied in N. tabacum collected from 2 populations growing in Botanical Gardens of Hindu College (HC) and Delhi University (DU). The meiotic studies revealed the presence of $2 n=48$. Although the 48 chromosomes resolved into 24 perfect bivalents at diakinesis stage in both the sex cells i.e. EMCs and PMCs (Figs. 1-4) in the 2 populations, they varied with respect to the nature of pairing and frequency of chiasmata. While male meiosis was studied in 40 PMCs of each population, female meiotic studies were carried out in 23 and 25 EMCs in the 2 i.e. $\mathrm{HC}$ and DU populations, respectively. The bivalents in PMCs appeared larger (Fig. 1) than the bivalents in EMCs (Fig. 4) and were tightly coiled showing a higher chiasmata frequency. The bivalents 
in EMCs either appeared in clumps (Fig. 2) or else showed overlapping (Fig. 3). However, wherever well separated bivalents were observed, they were mostly ring shaped (Fig. 4) with occasional cell showing 1-2 open rings or rod bivalents. While the chiasmata frequency in the EMCs of $\mathrm{HC}$ and DU collection works out to be $52.3 \pm 3.03$ and $53.4 \pm 2.86$, in the PMCs $61.05 \pm 2.67$ and $60.33 \pm 3.24$ chiasmata were recorded per cell in the 2 populations, respectively. The female sex cells showed 14.33 and $11.48 \%$ reduction in the chiasmata frequency. Statistical tests like students $t$-test and Fischer's variance ratio test (F-test) revealed the variations in the chiasmata frequency of the 2 sex cells to be significant.

\section{Discussion}

The behavior of chromosomes in the 2 sex cells is not always the same. Our earlier studies on male and female meiosis in different species have revealed variations in the behavior of chromosome with respect to frequency, distribution and position of chiasmata (Koul et al. 1995, 1999, 2000, Koul and Raina 1996, Koul

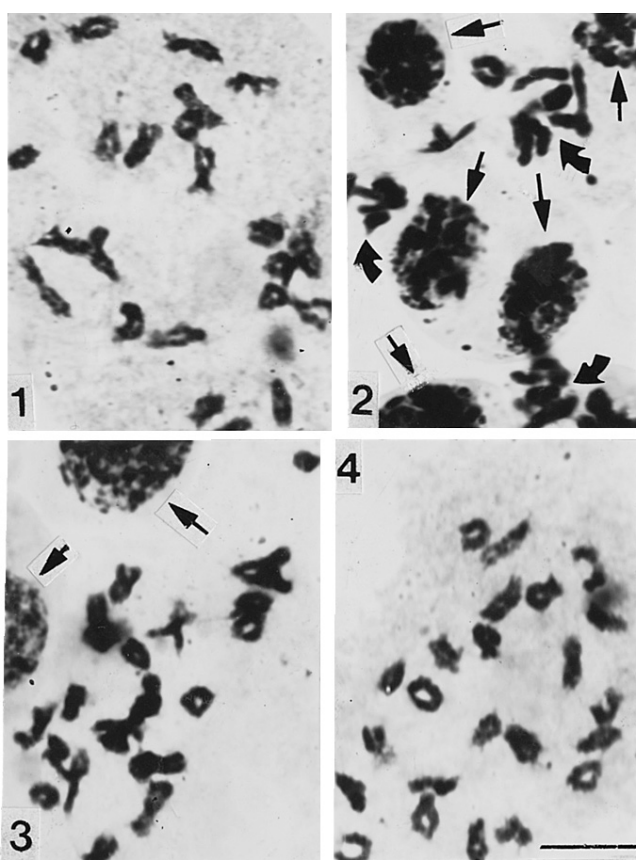

Figs. 1-4. Male and female meiosis in Nicotiana tabacum. 1) PMC at diakinesis showing 24 bivalents. 2) EMC showing bivalents in clumps (curved arrows) and scattered nucellar tissue (straight arrows). 3) EMC showing overlapping of bivalents with nucellar tissue (at arrows). 4) EMC with 24 bivalents. Scale: $10 \mu \mathrm{m}$. and Nagpal 2002). This study in Nicotiana tabacum has added one more species to the list of those showing chromosomal variations particularly with respect to chiasma frequency with male sex cells showing a higher frequency. Prior to this similar observations were made in Allium cepa, A. nigrum (Gohil and Kaul 1980), A. pallens, A. paniculatum, A. ursinum (Ved Brat 1966), Brassica oxyrrhina (Koul et al. 1995), Phlox drummondii (Koul and Raina 1996), Vicia johannis, V. narbonensis, V. hyaeniscyamus, V. galelaea (Koul et al. 1999). Variations in chiasmata frequency, which is a corollary of crossing over, point towards differential contribution of 2 sex cells towards generating variability. Since various events of meiosis are genetically controlled (Rees 1961, Baker et al. 1976) one may be tempted to conclude the varying behavior of chromosomes in 2 sex cells to be the result of 2 genetic systems/controls operational in the 2 sex cells. However, realizing the fact that several environmental agents can also affect crossing over and chiasma formation, some directly, some by affecting the degree of heterochromatinization (Sybenga 1975, 1992), it may also be concluded that the 2 sex cells might be having single genetic control which manifest 2 different effects due to different environmental conditions prevailing in the 2 sex cells. Whatever be the mechanism involved, it is well established that the 2 sex cells can vary drastically in the behaviour of chromosomes.

Comparative studies of male and female meiosis is of considerable practical interest. Besides giving a measure of the recombination level as mentioned elsewhere, female meiotic studies can be instrumental in discovering or isolating plants showing parthenogenesis or apomictic mode of reproduction. For instance meiotic studies in Allium tuberosum (Gohil and Kaul 1981) and its close relative A. odorum (Häkansson and Levan 1957) showed endoduplicational meiosis in female sex cells which had been instrumental in parthenogenetic development of seeds (Gohil and Kaul 1981). Similarly, in members of families like Asteraceae, Poaceae, Rutaceae, Rosaceae, Liliaceae and Or- 
chidaceae (Sybenga 1992) studies of female meiosis and/or development of female gametophyte has revealed the presence of apomicts. Since tremendous amount of work is being done to induct apomixis in elite hybrid plants with a view to produce elite clonal seeds in which the benefit of hybrid vigour is maintained and passed on to progeny (Maheshwari et al. 1998), the study of female meiosis will be of tremendous help. This is so because the commonest way for the origin of apomict is the failure of meiosis. If apomicts are isolated it will facilitate the process of isolation of important gene(s) responsible for inducing apomixis in plants. Therefore, a large number of plants need to be studied for both male and female meiosis not only for isolating apomicts but also for understanding the genetic/breeding system operational in them.

\section{Acknowledgements}

KKK gratefully acknowledges the financial assistance from DST.

\section{References}

Baker, B. S., Carpenter, A. T. C., Esposito, M. S., Esposito, E. and Sandler, L. 1976. The genetic control of meiosis. Ann. Rev. Genet. 10: 53-134.

Bennett, M. D., Finch, R. A., Smith, J. B. and Rao, M. K. 1973. The time duration of female meiosis in rye, wheat and barley. Proc. Roy. Soc. Lond. B 183: 301-319.

Cameron, D. R. 1959. The monosomics of Nicotiana tabacum. Tobacco Sci. 3: 164-166.

Clausen, R. E. and Cameron, D. R. 1944. Inheritance of Nicotiana tabacum. XVIII. Monosomic analysis. Genetics 29: $447-477$.

Davies, E. D. G. and Jones, G. H. 1974. Chiasma variation and control in pollen mother cells and embryo-sac mother cells of rye. Genet. Res. 23: 185-190.

Fogwill, M. 1958. Difference in crossing over and chromosome size in sex cells of Lilium and Fritillaria. Chromosoma 77: $123-127$.

Gerstel, D. U. and Burns, J. A. 1976. Enlarged euchromatic chromosomes (megachromosome) in hybrids between Nicotiana tabacum and N. plumbaginifolia. Genetica 46: 139-153.

Gohil, R. N. and Kaul, R. 1980. Studies on male and female meiosis in Indian Allium. I. Four diploid species. Chromosoma 17: 123-127.

— and - 1981. Studies on male and female meiosis in Indian Allium. II. Autotetraploid Allium tuberosum. Chromosoma 82: 735-739.

Häkansson, A. and Levan, A. 1957. Endoduplicational meiosis in Allium odorum. Hereditas 43: 179-200.

Koul, A. K. 1983. Two track heredity in hermaphrodite angiosperms. Phytomorphology 33: 137-142.

Koul, K. K. and Gohil, R. N. 1989. Male and female meiosis in Calamogrostis stoliczkai Hook. Ind. J. Cytol. Genet. 24: $164-166$.

- and Nagpal, R. 2002. Sex incidences of chiasmata variation in respect of position, distribution and frequency in some important legumes and grasses. Caryologia 55: 249-259.

—, - and Raina, S. N. 1995. Differential chromosome behaviour in the male and female sex cells of Brassica oxyrrhina Coss (Brassicaceae). Caryologia 48: 335-339.

- - - and Sharma, A. 2000. Chromosome behaviour in the male and female sex mother cells of wheat (Triticum aestivum L.), oat (Avena sativa L.) and pearl millet (Pennisetum americanum (L.) Leeke). Caryologia 53: 175-183.

— and Raina, S. N. 1996. Male and female meiosis in diploid and colchitetraploid Phlox drummondii Hook. (Polemoniaceae). Bot. J. Linn. Soc. 122: 243-251.

—, - , Parida, A. and Bisht, M. S. 1999. Sex differences in meiosis between Vicia faba and its close wild relatives. Bot. J. Linn. Soc. 129: 239-247.

Maheshwari, S. C., Maheshwari, N., Khurana, J. P. and Sopory, S. K. 1998. Engineering apomixes in crops; A challenge for plant molecular biologist in the next century. Current Science 75: 1141-1147.

Reed, S. M. 1991. Cytogenetic evolution and aneuploidy in Nicotiana. In: Tuschiya, T. and Gupta, P. K. (eds.). Chromosome Engineering in Plants. Genetics, Breeding, Evolution. Part B. Elsevier. New York. 1-630.

Rees, H. 1961. Genotypic control of chromosome form and behaviour. Bot. Rev. 27: 288-318.

Smith, H. H. 1979. The genus as a genetic resource. In: Durbin, R. D. (ed.). Nicotiana: Procedures for Experimental Use. USDA Technical Bulletin No. 1586. 1-16.

Sybenga, J. 1975. Meiotic configurations. Springer. Berlin, Heidelberg, New York. 
- 1992. Cytogenetics in plant breeding. Springer Verlag Press. Berlin. pp. 4681.

Ved Brat, S. 1966. Genetic systems in Allium. II. Sex differences in meiosis. In: Darlington C. D. and Lewis, K. R. (eds.). "The Chromosomes Today". Oliver and Boyd, London. 31-49.

Vosa, C. G. 1972. Two track heredity. Differentiation of male and female meiosis in Tulbaghia. Caryologia 25: 275-281. 